\title{
Uma conversa com Shulman e Vergnaud: reflexões teóricas de uma metassíntese qualitativa
}

\author{
Edvonete Souza de Alencar*
}

\section{Resumo}

Este artigo traz reflexões teóricas desenvolvidas em uma Tese que investigou os referenciais teóricos que sustentam pesquisas brasileiras, publicadas entre 1997 e 2015, relativas à formação contínua de professores dos anos iniciais do Ensino Fundamental sobre o Campo Conceitual Multiplicativo. A metodologia utilizada para sua realização foi a metassíntese qualitativa. A busca pelas investigações ocorreu a partir do site do Banco de Teses da Capes, mediante uso de expressões relacionadas ao objetivo do estudo, no qual selecionamos 27 pesquisas. Com este grupo buscamos os referenciais teóricos mais citados pelas investigações e observamos que foram: Shulman e Vergnaud. Encontramos nove pesquisas que utilizaram os dois referenciais teóricos. Com este grupo menor de investigações realizamos a metassíntese qualitativa. Os resultados indicam a importância do conhecimento específico do conteúdo (Shulman ) contribuindo ao professores para a análise da resolução dos alunos e a importância do esquema nas atividades de ensino e aprendizagem (Vergnaud).

Palavras-Chave: estudos teóricos; ensino e aprendizagem; formação de professores

\section{Conversation with Shulman and Vergnaud: theo- retical reflections of a qualitative meta-synthesis}

\section{Abstract}

This article brings theoretical reflections developed in a Thesis that investigated the theoretical references that support Brazilian research, published between 1997 and 2015, concerning the continuous formation of

* Doutora em Educação Matemática - UFGD 
teachers of the initial years of Elementary Education on the Multiplicative Conceptual Field. The methodology used for its accomplishment was the qualitative metasynthesis. The search for the investigations occurred from the site of the Bank of Thesis of Capes, using expressions related to the purpose of the study, in which we selected 27 surveys. With this group we look for the theoretical references most cited by the investigations and we observe that they were: Shulman and Vergnaud. We found nine studies that used the two theoretical references. With this smaller group of investigations we performed the qualitative meta-synthesis. The results indicate the importance of specific knowledge of content (Shulman) contributing to the teachers for the analysis of the students' resolution and the importance of the scheme in teaching and learning activities (Vergnaud).

Keywords: theoretical studies; teaching and learning; teacher training

\section{Una conversación con Shulman y Vergnaud: re- flexiones teóricas de una metassíntesis cualitativa}

\section{Resumen}

Este artículo trae reflexiones teóricas desarrolladas en una Tesis que investigó los referentes teóricos que sustentan investigaciones brasileñas, publicadas entre 1997 y 2015, relativas a la formación continua de profesores de los años iniciales de la Enseñanza Fundamental sobre el Campo Conceptual Multiplicativo. La metodología utilizada para su realización fue la metassíntesis cualitativa. La búsqueda por las investigaciones ocurrió a partir del sitio del Banco de Tesis da Capes, mediante el uso de expresiones relacionadas con el objetivo del estudio, en el que seleccionamos 27 investigaciones. Con este grupo buscamos los referentes teóricos más citados por las investigaciones y observamos que fueron: Shulman y Vergnaud. Encontramos nueve investigaciones que utilizaron los dos referenciales teóricos. Con este grupo menor de investigaciones realizamos la metassíntesis cualitativa. Los resultados indican la importancia del conocimiento específico del contenido (Shulman) contribuyendo al profesorado para el análisis de la resolución de los alumnos y la importancia del esquema en las actividades de enseñanza y aprendizaje (Vergnaud). Palabras clave: estudios teóricos; enseñanza y aprendizaje; formación de professores 


\section{Introdução}

Este artigo traz um excerto de uma Tese, que desenvolveu uma metassíntese qualitativa dos referenciais teóricos que sustentam pesquisas brasileiras publicadas no período de 1997 a 2012 a respeito da formação contínua de professores dos anos iniciais do Ensino Fundamental sobre o Campo Conceitual Multiplicativo.

A problemática de nossa investigação iniciou com as leituras de algumas investigações intituladas estados da arte $^{1}$, como: André (2002) e Brzezinski (2006), que desenvolveram investigações de mapeamento sobre a formação de professores. Notamos que não foi abordado por estas investigações a formação de professores de Matemática.

Além dessas investigações, estudamos a dissertação de Melo (2006) intitulada "Três décadas de pesquisas em Educação Matemática na Unicamp: um estudo histórico a partir de teses e dissertações da Unicamp no período de 1976 a 2003". A autora apresenta um estudo sobre a formação de professores em Matemática, mas restringe-se aos dados da Unicamp e focaliza sobre os aspectos históricos e identifica o movimento das pesquisas da Educação Matemática. Lemos também o artigo de Melo e Fiorentini (2008), que mencionam o aumento do número de estudos sobre formação de professores que ensinam Matemática e nos faz perceber a existência de "diversidade teórica" (MELO e FIORENTINI, 2008, p. 6) E revelam a necessidade de estudos sobre essa temática.

Diante do exposto, observamos que os referenciais teóricos são a base das investigações e que até então não haviam realizado uma metassíntese qualitativa em outros estudos.

Com isso, nosso objetivo foi investigar os referenciais teóricos que sustentam pesquisas brasileiras, publicadas entre 1997 a 2015, a respeito da formação contínua de professores dos anos iniciais do Ensino Fundamental sobre o Campo Conceitual Multiplicativo.

Segundo Romanowski e Ens (2006, p.39), estados da arte "recebem essa denominação quando abrangem toda uma área do conhecimento nos diferentes aspectos que geraram produções". 
Para isso, o questionamento que norteou nossa investigação foi: "Há semelhanças, diferenças e complementariedades na maneira como as pesquisas empregaram as ideias originais dos referenciais teóricos?

A partir do que foi apresentado, organizamos a metodologia de pesquisa a ser utilizada e seus respectivos procedimentos.

\section{Metassíntese qualitativa: caminhos metodológicos}

A metassíntese qualitativa é uma das modalidades de pesquisa que realizam uma revisão sistemática de investigações qualitativas. Segundo Vosgerau e Romanowski (2014), há duas modalidades metodológicas: as revisões que mapeiam, que são os estados da arte e os estados do conhecimento, e as que avaliam e sintetizam, como é o caso da metanálise e da metassíntese.

A metassíntese qualitativa possui origem de pesquisas internacionais da área da saúde. A Fundação Cochrane em meados de 1998 estabeleceu algumas orientações e formações metodológicas que permitiu o uso dessa metodologia por outras áreas.

Assim, Matheus (2009) define a metassíntese qualitativa como "integração interpretativa de achados qualitativos (derivados de estudos fenomenológicos etnográficos, da teoria fundamentada dos dados e outros), que são a síntese interpretativa dos dados" (p.544). Para a autora os dados e as relações entre eles permitem que se desenvolva uma nova interpretação. Portanto, essa análise das pesquisas não se torna meramente um resumo dos dados, mas uma nova construção de conhecimento utilizando-se os dados primários que são as dissertações e teses.

Em consonância a Matheus (2009), o autor Fiorentini (2013) nos diz que a metassíntese qualitativa:

visa produzir interpretações ampliadas de resultados ou achados de estudos qualitativos obtidos por estudos primários (como são as dissertações, teses e pesquisas de professores), os quais são selecionados atendendo a um interesse específico do pesquisador acerca de um fenômeno a ser investigado e/ ou teorizado (FIORENTINI, 2013, p.78) 
Há menção do autor sobre a utilização das interpretações dos autores sobre as dissertações e teses, o que este chama de metainterpretação, nos dá os procedimentos necessários para que seja realizada a metassíntese qualitativa.

Assim para a realização, da metassíntese qualitativa, nossa busca envolveu a apresentação das origens (instituições e regiões em que foram publicadas) e os fichamentos (título da dissertação/tese, autor, instituição, no de defesa, número de páginas, palavras-chave, resumo, objetivo, referencial teórico, análises e referenciais bibliográficos).

Nossa busca foi realizada no site do Banco de Teses da Capes, no dia 23 de julho de 2016. Para isso, utilizamos as expressões "Formação de Professores" e "Campo Conceitual Multiplicativo" em títulos, resumos ou palavras-chave, nos programas de Educação e Educação Matemática, com área de concentração em Educação Matemática, o que resultou em 329 dissertações e teses. Por meio dos resumos, selecionamos as investigações que mencionavam "Formação Contínua e Anos Iniciais” e encontramos 27 pesquisas. As pesquisas são: Canoas (1997), Ewbank (2002), Araújo (2003), Gregolon (2005), Santos (2005), Arrais (2006), Canova (2006), Campos (2007), Soares (2007), Garcia Silva (2007), Vasconcelos (2008), Nurnberg (2008), Neves (2008), Camejo silva (2009), Gomes (2010), Cervantes (2011), Rocha (2011), Santana (2011), Carvalho (2012), Alencar (2012), Merlini (2012), Alencar (2012), Santos (2012), Oliveira (2014), Souza(2015), Silva Junior (2015), Rogeri (2015).

Estamos cientes de que nesta Tese não abarcamos todas as pesquisas realizadas no país, pois esse tipo de estudo é sempre inconcluso porque o período da busca é finito e depende do que os autores declararam como foco da pesquisa, das palavras-chave, do título e do resumo.

Para obter uma visão do que abarcou as 27 pesquisas, realizamos breves fichamentos das investigações. Com essas investigações realizamos o procedimento arrazoado bibliográfico no qual observamos a frequência de citações e/ou menções que apareciam pelo menos nas análises e na problemática (e/ou quadro 
teórico) de cada uma das referências teóricas presentes nas vinte e duas dissertações e teses.

As obras que sustentam as pesquisas foram organizadas em uma tabela por autor e número de menções e/ou citações. Mas nesse artigo apresentamos o gráfico com os referenciais teóricos mais utilizados pelas investigações.

Gráfico 1 - Frequência total das obras teóricas mencionadas el ou citadas nas 27 pesquisas.

\section{USO DOS REFERENCIAIS TEÓRICOS}

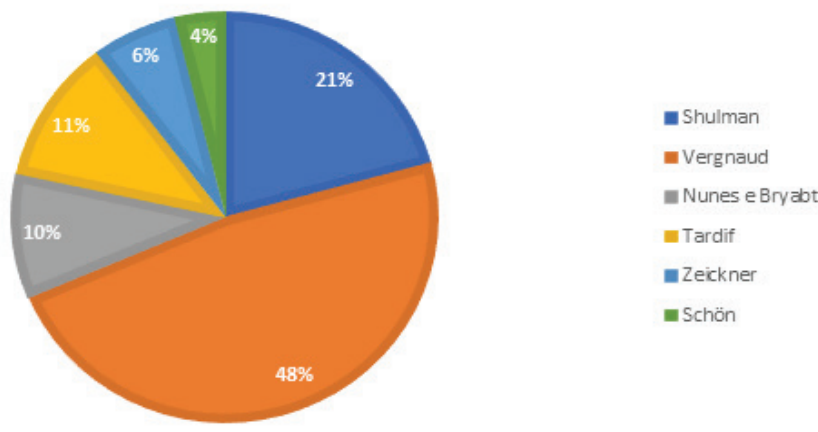

Do gráfico pode-se observar que os autores que possuem mais obras teóricas mencionadas e/ou citadas são Vergnaud e Shulman, seguidos por Tardif, Nunes e Bryant, Schön e Zeichner.

Essa busca por referenciais teóricos que sustentaram as pesquisas foi elaborada a partir de leituras e da contagem das menções e / ou citações em dissertações e teses selecionadas criteriosamente. Essa contagem ocorreu na problemática e/ou quadro teórico e nas análises. Por essas características, esse procedimento constituiu-se em um arrazoado bibliográfico.

Com isso, para realizar nossa metassíntese qualitativa, buscamos selecionar as pesquisas que utilizam Vergnaud e Shulman. Identificamos nove pesquisas que são apresentadas na tabela a seguir: 
Tabela 1 - Grupo de nove pesquisas selecionadas

\begin{tabular}{|c|c|c|c|c|c|}
\hline Título & Autor & Instit. & $\mathrm{D} / \mathrm{T}^{*}$ & Ano & $\begin{array}{l}\text { Ori- } \\
\text { gem }\end{array}$ \\
\hline $\begin{array}{l}\text { O conceito de fração em seus diferentes } \\
\text { significados: um estudo diagnóstico } \\
\text { junto a professores que atuam no Ensi- } \\
\text { no Fundamental }\end{array}$ & $\begin{array}{l}\text { SANTOS, } \\
\text { Aparecido }\end{array}$ & PUCSP & $\mathrm{D}$ & 2005 & SP \\
\hline $\begin{array}{l}\text { As dificuldades na aprendizagem da } \\
\text { divisão: análise da produção de erros } \\
\text { dos alunos do Ensino Fundamental } \\
\text { e sua relação com o ensino praticado } \\
\text { pelos professores }\end{array}$ & CAMPOS, E. & $\mathrm{UCDB}$ & $\mathrm{D}$ & 2007 & MS \\
\hline $\begin{array}{l}\text { O desafio do desenvolvimento pro- } \\
\text { fissional docente: análise da formação } \\
\text { continuada de um grupo de professores } \\
\text { das séries iniciais do Ensino Fundamen- } \\
\text { tal, tendo como objeto de discussão o } \\
\text { processo de ensino e aprendizagem das } \\
\text { frações }\end{array}$ & $\begin{array}{l}\text { GARCIA } \\
\text { SILVA, A. }\end{array}$ & PUCSP & $\mathrm{T}$ & 2007 & $\mathrm{SP}$ \\
\hline $\begin{array}{l}\text { A formação docente e o ensino de pro- } \\
\text { blemas combinatórios: diversos olhares, } \\
\text { diferentes conhecimentos }\end{array}$ & ROCHA, C. & UFPE & $\mathrm{D}$ & 2011 & $\mathrm{PE}$ \\
\hline $\begin{array}{l}\text { Conhecimento profissional docente de } \\
\text { professores do } 5 .^{\circ} \text { ano em uma escola } \\
\text { com bom desempenho em Matemática: } \\
\text { o caso das estruturas multiplicativas }\end{array}$ & ALENCAR, E & UNIBAN & $\mathrm{D}$ & 2012 & $\mathrm{SP}$ \\
\hline $\begin{array}{l}\text { As potencialidades de um processo } \\
\text { formativo para a reflexão na e sobre } \\
\text { a prática de uma professora das séries } \\
\text { iniciais: um estudo de caso. }\end{array}$ & MERLINI, V. & PUCSP & $\mathrm{T}$ & 2012 & $\mathrm{SP}$ \\
\hline $\begin{array}{l}\text { Processos de formação colaborativa } \\
\text { com foco no Campo Multiplicativo: } \\
\text { um caminho possível com professoras } \\
\text { polivalentes }\end{array}$ & $\begin{array}{l}\text { SANTOS, } \\
\text { Aparecido }\end{array}$ & PUCSP & $\mathrm{T}$ & 2012 & $\mathrm{SP}$ \\
\hline $\begin{array}{l}\text { Raciocinio combinatório na resolução } \\
\text { de problemas nos anos iniciais do } \\
\text { ensino fundamental: um estudo com } \\
\text { professores }\end{array}$ & OLIVEIRA,E. & PUCSP & $\mathrm{D}$ & 2014 & $\mathrm{SP}$ \\
\hline $\begin{array}{l}\text { Intervenções didáticas no Ensino de } \\
\text { Frações e as formas de professores }\end{array}$ & $\begin{array}{l}\text { SILVA JU- } \\
\text { NIOR, F. }\end{array}$ & UNIAN & $\mathrm{D}$ & 2015 & $\mathrm{SP}$ \\
\hline
\end{tabular}

* Para as siglas: D - Dissertação, T - Tese 
Com essas investigações realizamos a metassíntese qualitativa sobre os referenciais de Shulman e Vergnaud, formando categorias de semelhança e complementariedades.

\section{Metassíntese qualitativa de Shulman}

Nessa seção buscamos formar categorias sobre o uso do referencial teórico de Shulman. Essas categorias surgiram do estudo dos fichamentos dessas pesquisas por meio de semelhanças e possíveis complementariedades do modo como utilizaram as obras desse autor.

Para metassintetizar o uso dos referenciais teóricos de Shulman pelas nove pesquisas, realizamos uma leitura, na íntegra, das dissertações e teses; em seguida, procedemos aos fichamentos, nos quais identificamos as categorizações. Observamos ainda o que as pesquisas utilizaram da obra de Shulman por meio dos textos originais. Por isso, apresentamos inicialmente algumas considerações sobre os estudos do autor utilizados nas investigações e, a seguir, as seis categorizações encontradas.

I. Focaliza os três primeiros conhecimentos (específico do conteúdo, pedagógico e curricular), definindo-os.

II. Denomina os sete conhecimentos definidos por Shulman, mas não os define.

III. Ressalta a importância do conhecimento específico do conteúdo.

IV.Complementa as ideias de Shulman com relação ao conhecimento pedagógico do conteúdo, contribuindo para a formação profissional docente.

V. Complementa as ideias de Shulman com relação ao conhecimento específico do conteúdo, contribuindo para a formação dos docentes em Matemática.

VI. Traz à luz aspectos pouco abordados da teoria nos estudos de Shulman.

O quadro a seguir sintetiza as categorias (I, II, III, IV, V e VI) obtidas das comparações feitas entre as pesquisas de Santos (2005), 
Campos (2007), Garcia Silva (2007), Rocha (2011), Alencar (2012), Merlini (2012), Santos (2012), Oliveira (2014) e Silva Junior (2015).

\section{Quadro 1- Categorias relativas ao emprego dos estudos de Shulman}

\begin{tabular}{|l|l|l|l|l|l|l|l|l|}
\hline $\begin{array}{l}\text { Santos } \\
(2005)\end{array}$ & $\begin{array}{l}\text { Campos } \\
(2007)\end{array}$ & $\begin{array}{l}\text { Garcia } \\
\text { Silva } \\
(2007)\end{array}$ & $\begin{array}{l}\text { Rocha } \\
(2011)\end{array}$ & $\begin{array}{l}\text { Alencar } \\
(2012)\end{array}$ & $\begin{array}{l}\text { Merlini } \\
(2012)\end{array}$ & $\begin{array}{l}\text { Santos } \\
(2012)\end{array}$ & $\begin{array}{l}\text { Oliveira } \\
(2014)\end{array}$ & $\begin{array}{l}\text { Silva } \\
\text { Junior } \\
(2015)\end{array}$ \\
\hline I & I & I & & I & & & I & I \\
\hline II & & & II & II & II & & & \\
\hline & III & III & & & & III & & III \\
\hline & IV & IV & IV & & & & & \\
\hline & & V & & & & & & \\
\hline & VI & VI & VI & VI & & VI & & \\
\hline
\end{tabular}

Neste artigo apresentamos as reflexões da Categoria III que ressalta a importância do conhecimento específico do conteúdo.

Ao realizar a metassíntese qualitativa observamos que Campos (2007), Garcia Silva (2007), Santos (2012) e Silva Jr (2015) apresentam, nas análises de suas investigações, a importância de o docente ter pleno domínio do conteúdo a ser ensinado para que possa desenvolver os outros dois conhecimentos citados por Shulman (1987): pedagógico do conteúdo e curricular. Como podemos notar nos trechos a seguir:

Durante o exercício profissional, os professores acabam construindo um novo tipo de conhecimento da área específica, que é melhorado e enriquecido por outros tipos de conhecimentos. Esse conhecimento, específico da docência é denominado conhecimento didático do conteúdo. É uma combinação entre o conhecimento da disciplina e o conhecimento do "modo de ensinar" e de tornar a disciplina compreensível para o aluno. [...] ( CAMPOS, 2007, p. 59)

Consideramos, assim como Shulman (1986), que o domínio do conhecimento do conteúdo é importante nos processos de aprendizagem docente e, se ele não vem ocorrendo a contento, é necessário que haja, na formação inicial, um enfoque mais amplo do conceito de números racionais, complementado por uma análise dos diferentes significados de sua 
representação fracionária. É importante também chamar a atenção para a necessidade de buscar o isomorfismo entre a formação recebida pelo professor e o tipo de educação que dele será exigida. É preciso haver certa coerência entre o conhecimento didático do conteúdo e a forma como esse conhecimento se processa. Acreditamos que, na formação de professores, uma das principais fontes de aprendizagem é o método por meio do qual conhecimentos profissionais são tratados junto aos professores, pois os docentes são também "modelos de professor". ( GARCIA SILVA, 2007,p.270)

Os professores precisam saber o conteúdo acadêmico pelo qual são responsáveis por ensinar e como transformá-lo, a fim de conectá-lo com aquilo que os estudantes já sabem para o desenvolvimento de uma compreensão mais elaborada. (SANTOS, 2012, 65)

A partir de 1983, Shulman, por meio da observação desse tipo trabalho, percebeu que havia um ponto importante a ser considerado. Nesse âmbito, o ensino ainda era considerado como uma atividade geral e sem relação com o que o próprio professor sabia, individualmente. Assim, para simplificar as complexidades do ambiente escolar, os trabalhos de pesquisa, até aquele momento, ignoraram como o conteúdo específico era transformado a partir do conhecimento que o professor tinha; tampouco, consideravam como as formas particulares de ensino influenciavam no que os estudantes passavam a conhecer corretamente ou de forma equivocada. Shulman iniciou então o projeto "Knowledge Growth in a Profession", focando a realização de trabalhos que contemplassem esses aspectos, até então esquecidos, aos quais ele chamou de "paradigma perdido". ( SILVA JUNIOR, 2015, p41-42)

A respeito disso, Shulman (1987) menciona que em estudos anteriores ele já pesquisava sobre a importância e a necessidade de os docentes terem domínio do conteúdo para o ensino da disciplina e também como este influenciava nos outros conhecimentos necessários para a docência. Segundo Shulman (1987) esses conhecimentos são: 1) Específico do conteúdo, 2) Pedagógico do conteúdo, 3) Curricular, 4) Pedagógico geral, 5) Dos alunos e suas características, 6) Dos contextos educativos e 7) Dos objetivos, das finalidades, dos valores educativos e dos fundamentos filosóficos e históricos -, mas não os aprofundam, como notamos nos trechos selecionados. Com isso, de modo geral todos se relacionam. 
Assim, podemos compreender quais aspectos foram mais abordados e quais poderiam ser mais mencionados.

Metassíntese qualitativa de Vergnaud

Dos dados das pesquisas, emergiram categorias sobre os elementos explicitados da Teoria dos Campos Conceituais (TCC) e do Campo Conceitual Multiplicativo. Aqui apresentamos oito categorias relativas ao seu emprego pelas investigações.

I. Apresenta a definição da Teoria dos Campos Conceituais.

II. Considera um campo conceitual como um conjunto de situações propostas aos estudantes.

III. Interpreta a noção de esquema na Teoria dos Campos Conceituais.

IV. Considera o esquema como organização invariante em uma classe de situações.

V. Apresenta a formação de conceitos pela terna de conjuntos: S - Situações; I - Invariantes; e R - Representações simbólicas.

VI. Dá grande atenção à construção de conceitos matemáticos.

VII. Detalha a apresentação do Campo Conceitual Multiplicativo.

VIII. Traz noções sobre o Campo Conceitual Aditivo (cálculo relacional e cálculo numérico) para o Campo Conceitual Multiplicativo.

O quadro 2 a seguir relaciona as pesquisas com as categorias relativas ao emprego da Teoria de Vergnaud. 
Quadro 2 - Categorias relativas ao emprego da Teoria de Vergnand

\begin{tabular}{|l|l|l|l|l|l|l|l|l|}
\hline $\begin{array}{l}\text { Santos } \\
(2005)\end{array}$ & $\begin{array}{l}\text { Campos } \\
(2007)\end{array}$ & $\begin{array}{l}\text { Garcia } \\
\text { Silva } \\
(2007)\end{array}$ & $\begin{array}{l}\text { Rocha } \\
(2011)\end{array}$ & $\begin{array}{l}\text { Alencar } \\
(2012)\end{array}$ & $\begin{array}{l}\text { Merlini } \\
(2012)\end{array}$ & $\begin{array}{l}\text { Santos } \\
(2012)\end{array}$ & $\begin{array}{l}\text { Oliveira } \\
(2014)\end{array}$ & $\begin{array}{l}\text { Silva } \\
\text { Junior } \\
(2015)\end{array}$ \\
\hline I & I & I & I & I & I & I & I & I \\
\hline II & II & II & II & II & II & II & II & II \\
\hline III & III & III & & III & III & III & III & III \\
\hline IV & IV & IV & IV & IV & IV & IV & IV & \\
\hline V & V & V & V & V & V & V & V & \\
\hline & & VI & VI & & VI & VI & & \\
\hline & & & & VII & VII & VII & & \\
\hline & & & & VIII & & & & \\
\hline
\end{tabular}

Neste artigo apresentamos a Categoria III que traz a interpretação dos autores sobre a noção de esquema na Teoria dos Campos Conceituais.

Campos (2007), Garcia Silva (2007), Alencar (2012), Merlini (2012) e Santos (2012) baseiam-se na organização dos invariantes (esquema) e nas classes de situações de um campo conceitual, como mostrado por Vergnaud.

As pesquisas caracterizam o esquema as ações desenvolvidas pelos alunos nas situações. Serve, portanto, de base para a construção dos conhecimentos que ocorrem nessa relação evidenciando os conhecimentos operatórios envolvidos.

Santos (2005) relata que "o esquema se refere à forma estrutural da atividade, isto é, diz respeito à organização das invariantes da atividade pelo estudante em uma classe de situações dadas”. (SANTOS, 2005, p.29)

Garcia Silva (2007) enfatiza que os esquemas revelam os conhecimentos em ação presentes nas situações-problema, "os elementos cognitivos que fazem com que a ação do sujeito seja operatória". (GARCIA SILVA, 2007, p. 78)

Campos (2007) confirma as citações anteriores e menciona as relações existentes entre as situações e o sujeito; acrescenta que é por meio dessas interações que os esquemas vão se constituindo. 
Vergnaud (1990) aprofunda a noção de esquema desenvolvida por Piaget, tendo como um dos pressupostos básicos que o conhecimento se constitui e se desenvolve ao longo de um período de tempo e a partir da interação adaptativa do sujeito com as situações que experiência. (CAMPOS, 2007, p.27)

As relações também são evidenciadas por Merlini (2012):

A noção de esquema é particularmente adaptada para designar e analisar classes de situações para as quais o sujeito dispõe em seu repertório, a um dado momento de seu desenvolvimento e sob certas circunstâncias, as competências necessárias ao tratamento relativamente imediato da situação. Muitos esquemas são evocados sucessivamente e mesmo simultaneamente em uma situação nova para o sujeito. (Merlini, 2012, p. 89-90)

Em consonância com o que já foi relatado, Alencar (2012) afirma ainda que o esquema contém "uma totalidade dinâmica funcional, uma função que toma seus valores de entrada e de saída, incorporando tempo e suas dimensões e uma organização invariante para uma situação”. (ALENCAR, 2012, p. 64)

Alencar (2012) menciona também quatro categorias de esquemas: "o objetivo; como a atividade é gerada; os invariantes operatórios representados pelo teorema em ação; e o conhecimento em ação, as antecipações ou inferências". (ALENCAR, 2012, p. 64)

Santos (2012) ressalta que o esquema:

é de suma importância para as duas classes de situações, pois é ele o responsável por organizar as ações do sujeito aprendiz frente às situações. No caso da primeira classe de situações, o sujeito aprendiz lança mão de um esquema que já possui e está internalizado, e que na maioria das vezes se torna automatizado. Já na segunda classe de situações, os esquemas de ação do sujeito aprendiz não são suficientemente adequados para um tratamento imediato de uma dada situação, desafiando-o à descoberta (com ou sem ajuda) de novos esquemas e novas formas de conduzir o surgimento desses esquemas. (SANTOS, 2012, p. 90) 
Corroborando as citações anteriores, Oliveira (2014, p.60) diz que "o esquema é uma organização feita pelo próprio indivíduo quando ele tem como objetivo conduzir o processo de resolução de uma classe de situação, porém não consegue expressá-la por meio da linguagem natural". Para subsidiar sua afirmação, a autora cita Vergnaud (2009, p. 21): “o esquema não organiza somente a consulta observável, mas também o pensamento subjacente".

Silva Junior (2015) conceitua ou exemplifica esquema:

esquemas de ação são os primeiros reflexos que a criança tem, tais como sugar ou pegar, ao passo que nos esquemas de representação já existe a capacidade de diferenciar o significante e o significado. Nesse caso, a criança passa a representar ações, situações e experiências por meio desses esquemas. Portanto, o sentido de uma situação ou de um significante para um indivíduo tem relação com os esquemas, os comportamentos e a organização que essa situação traz à tona no indivíduo. Quando uma pessoa se depara com um número fracionário, ela automaticamente se defronta com uma série de esquemas que a permite lidar com a ideia de fração. Podemos dizer, então, que o desenvolvimento de um vasto repertório de esquemas constitui o chamado "desenvolvimento cognitivo". (SILVA JUNIOR, 2015, 36)

Salientamos que apesar de mostrar esquemas que poderiam ser analisados por meio da Teoria de Vergnaud, Rocha (2011) não faz essa relação. Notamos também que a autora não apresenta uma definição de esquema. Pode-se notar que as interpretações dos autores são semelhantes ao que Vegnaud $(1990,1982)$ propõe, mas alguns deles aprofundam a compreensão de esquema e complementam a definição. Tal aspecto pode ser observado na citação anterior de Silva Junior (2015), que define esquema utilizando um exemplo. Vergnaud (1990) define esquema como:

"a organização invariante de uma conduta para uma classe de situação dada. É nos esquemas que se deve investigar os conhecimentos-em-ação do sujeito e decidir os elementos cognitivos que permitem a ação do sujeito ser operatória" (VERGNAUD, 1990, p.134, tradução nossa) 
Para explicar o esquema, o autor cita o exemplo do domínio da motricidade, que, para ser desenvolvida, envolve categorias de ordem espacial, temporal e mecânica. Afirma que as operações se automatizam progressivamente; por isso, a consciência das ações é que permite observar as particularidades das situações. A automatização fica evidente na organização das ações, mas algumas decisões conscientes podem ter uma outra organização.

No entanto, a automatização não impede que o sujeito conserve e controle as condições nas quais a operação é realizada. Como exemplo, o autor cita o algoritmo da adição de números decimais, no qual os alunos são capazes de ter diversas ações, como intercalar o número zero, elevar o número, tornar decimal o número. Assim, as ações mostram uma parte de automaticidade e uma parte de decisão consciente.

$\mathrm{O}$ autor adianta ainda que "os algoritmos são esquemas, e os esquemas são objetos do mesmo tipo lógico dos algoritmos" (VERGNAUD, 1990, p.138, tradução nossa). Falta ao esquema somente a afetividade, a decisão e a propriedade de efetuar com segurança os passos.

Cita ainda que os esquemas são frequentemente eficazes, mas nem sempre efetivos. Um exemplo é quando uma criança utiliza um esquema ineficaz para uma situação e a experiência a faz mudar o esquema ou modificá-lo.

\section{Algumas conclusões e considerações.}

Constatamos que os aspectos dos estudos de Shulman, na Categoria III "Ressalta a importância do conhecimento específico do conteúdo", são evidenciados pelas pesquisas de modo semelhante ao que Shulman $(1986,1987)$. Não encontramos, em nossa metassíntese, diferenças nas interpretações dos autores das pesquisas analisadas com relação ao estudo de Shulman. Notamos que as reflexões dos estudos de Shulman (1986) influenciaram as pesquisas, mas que os elementos dos estudos de Shulman (1987) ainda são evidenciados. 
Quanto à metassíntese qualitativa relativa ao uso do referencial teórico de Vergnaud, referente à Categoria III que traz a interpretação dos autores sobre a noção de esquema na Teoria dos Campos Conceituais, notamos que as pesquisas dão importância para a noção de esquema e uma das pesquisas faz um complemento utilizando exemplos para explicar esquema.

Concluímos que Shulman (1986) ao apresentar o conhecimento específico do conteúdo, nos traz reflexões sobre sua importância ao docente na análise de resolução dos alunos. E Vergnaud (1990) nos permite refletir sobre a importância do esquema no processo de ensino e aprendizagem dos alunos.

\section{Referências}

ALENCAR, E. S. Formação de professores sobre o Campo Conceitual Multiplicativo: referenciais teóricos em pesquisas. Tese de Doutorado, São Paulo: Pontifícia Universidade Católica de São Paulo. Brasil. 2016

ANDRÉ, M. D. Formação de professor no Brasil (1990 -1998) (Serie Estado do Conhecimento n.6 ed.). Brasília: MEC/ Inep /Comped.2002

BANCO DE TESES. Acesso julho de 2016, disponível em Capes: http://www. bancodeteses.capes.gov.br. 2016

BRZEZINSKI, I. Formação de profissionais da educação (1997-2002) (Vol. Série estado do conhecimento n.10). Brasilia: MEC/Inep/Comped. 2006

FIORENTINI, D. Investigação em Educação Matemática desde a perspectiva acadêmica e profissional: desafios e possibilidades de aproximação. Cuadernos de Investigación y Formación en Educación Matemática, 8 (11), 61-82.2013

MELO, M. V., \& FIORENTINI, D. A pesquisa brasileira sobre formação de professores que ensinam matemática: diversidades teórico-metodológicas; desafios epistemológicos e a busca de identidade. EBRAPEM, Encontro Brasileiro de Estudantes de Pós-graduação em Educação Matemática: Quais os próximos desafio em Educação Matemática.2008

ROMANOWSKI, J. P., \& ENS, R. T. As pesquisas denominadas do tipo estado da arte em Educação. Revista Diálogo Educacional, 6(19).2006 
SHULMAN, L. Those who understand : knowledge growth in teaching. Education Researcher, 15(2), 4-14.1986

SHULMAN, L.. S. Knowledge and teaching: foundations of the new reform. Educational Review, Harvard, v. 57, p. p.1-22, 1987.

VERGNAUD, G. La théorie des champs conceptuels. Récherches em Didactique des Mathematiques, 23(10), 133-170.1990

APÊNDICE 1: Relação de dissertações/teses do corpus de análise deste artigo

ALENCAR, E. S. Conhecimento Profissional Docente de Professores do $\mathbf{5}^{\circ}$ ano em uma escola com bom desempenho em Matemática: o caso das estruturas multiplicativas. Dissertação de Mestrado, São Paulo: Universidade Bandeirantes.Sao Paulo, Brasil.2012

CAMPOS, E. G. As dificuldades na aprendizagem da divisão: análise da produção dos erros dos alunos do ensino fundamental e sua relação com o ensino praticado pelos professores Dissertação (Mestrado em Educação) . Universidade Católica Dom bosco: Campo Grande.2007

MERLINI, V. L. As potencialidades de um processo formativo para a reflexão na sobre a prática de uma professora das séries iniciais: um estudo de caso Tese (Doutorado em Educação Matemática) ed.. Pontifícia Universidade Católica de São Paulo: São Paulo.Brasil.2012.

OLIVEIRA, E. G. Raciocínio combinatório na resolução de problemas nos anos iniciais do ensino fundamental:um estudo com professores. Dissertação (Mestrado em Educação Matemática) Pontifícia Universidade Católica de São Paulo: São Paulo.2014

ROCHA, C. A. A formação docente e o ensino de problemas combinatórios: diversos olhares, diferentes conhecimentos Dissertação (Mestrado em Educação). UFPE: Recife.Brasil. 2011

SILVA, A. F. G. O desafio do desenvolvimento profissional docente: análise da formação continuada de um grupo de professores das séries iniciais do ensino fundamental, tendo como objeto de discussão o processo do ensino e aprendizagem de frações. Tese (Doutorado em Educação Matemática) PUC-SP São Paulo. Brasil.2007

SANTOS, A. D. O conceito de fração em seus diferentes significados: um estudo diagnóstico junto a professores que atuam no Ensino Fundamental Dissertação (Mestrado em Educação Matemática) . PUCSP: São Paulo.Brasil.2005 
SANTOS, A. Processo de formação colaborativa com foco no Campo Multiplicativo: um caminho possível com professoras polivalentes. Tese (Doutorado em Educação Matemática) . PUCSP: São Paulo. Brasil.2012

SILVA JUNIOR , F. J. Intervenções didáticas no ensino de frações e a formação de professores. Dissertação (Mestrado em Educação Matemática) Universidade Anhanguera. São Paulo Brasil. 2015 